\title{
Policía predictiva en España. Aplicación y retos futuros.
}

\section{Predictive policing in Spain. Implementation and future challenges.}

\author{
José Luis González-Álvarez, Ph.D (1.4) \\ Jorge Santos-Hermoso, M.Sc (2) \\ Miguel Camacho-Collados, Ph.D (3)
}

(1) Secretaría de Estado de Seguridad, Gabinete de Coordinación y Estudios, Ministerio del Interior, Madrid, España.

(2) Departamento de Psicología Biológica y de la Salud, Facultad de Psicología, Universidad Autónoma de Madrid (UAM), Madrid, España.

(3) Consejo de Estado, Madrid, España.

(4) Instituto de Ciencias Forenses y de la Seguridad (ICFS), Universidad Autónoma de Madrid (UAM). Madrid, España.

Email de correspondencia: jlga@interior.es

\section{Resumen}

Las decisiones en materia de politicas públicas deben estar basadas en el análisis riguroso de los datos efectuado a través de métodos y procedimientos cientificos, para evitar tomar decisiones basadas en datos sesgados o creencias erróneas. El desarrollo de algoritmos y modelos predictivos aplicados al análisis del crimen, ha permitido la aparición de la policía predictiva, que analiza una gran cantidad de datos históricos de criminalidad para identificar patrones y estimar la probabilidad de que sucedan crímenes en el futuro, y para ayudar a esclarecer crímenes pasados. Esto ha hecho posible que desde los cuerpos policiales se potencie el enfoque preventivo frente al enfoque reactivo. A lo largo de este trabajo se expondrán algunas de las principales aplicaciones de la policía predictiva en España, y se analizarán en detalle los avances en los ámbitos de la Violencia de Género y el homicidio. El objetivo principal es resumir los principales estudios que se han desarrollado en los últimos años, destacar algunas de las limitaciones que puede tener la aplicación de este tipo de metodologias, y sobre todo señalar los beneficios que puede reportar la aplicación de modelos predictivos en la gestión de los recursos policiales.

\section{Palabras Clave}

Policía predictiva; Criminalidad; Homicidio; Feminicidio; Violencia de Género; Sistema VioGén; Veripol.

\begin{abstract}
Public policy decisions must be based on rigorous data analysis carried out through scientific methods and procedures to avoid making decisions based on biased data or erroneous beliefs. The development of algorithms and predictive models applied to crime analysis has allowed the emergence of predictive police, which analyzes a large amount of historical crime data to identify patterns and estimate the probability of future crimes, and to help a clarification of past crimes. Predictive policing has made it possible for the police forces to strengthen the preventive approach over the reactive approach. The current study explains some of the implementations of predictive policing in Spain. The advances in the areas of gender-based violence and homicide will be analyzed in detail. The main objective is to summarize the studies that have been developed in recentyears and the limitations and benefits of predictive methodologies.
\end{abstract}

\section{Keywords}

Predictive policing; Criminality; Homicide; Femicide; Gender-based violence; VioGén System; Veripol. 


\section{Policía predictiva}

Cada vez está más extendida la idea de que las decisiones, en cuanto a políticas públicas se refiere, deben estar basadas en el análisis riguroso de los datos efectuado a través de métodos y procedimientos científicos, evitando así tomar decisiones basadas en datos sesgados o creencias erróneas (Hart y Yohannes, 2019). Uno de los ámbitos gubernamentales más importantes, por cuanto afecta a los derechos y libertades fundamentales de las personas, es el que tiene que ver con la seguridad de los ciudadanos y la lucha contra el crimen. En este contexto, la acumulación, tratamiento y análisis de grandes cantidades de datos (big data), ha permitido llegar a lo que actualmente se conoce como policía predictiva.

Aunque no existe una definición única de este concepto, entre todas las propuestas se pueden identificar una serie de características clave (Meijer y Wessels, 2019). Así, se entiende que lo que caracteriza a la policía predictiva es el uso de una gran cantidad de datos históricos, que se analizan mediante técnicas cuantitativas, para estimar a través de algoritmos un valor desconocido (Provost y Fawcett, 2013), y cuyos resultados ayudarán a gestionar de forma más eficaz los recursos policiales, como por ejemplo, la distribución de las patrullas (Bennett Mosses y Chan, 2016; Camacho-Collados y Liberatore, 2015; Hardyns y Rummens, 2017; Perrot, 2017; Perry et al., 2013; Tayebi y Glässer, 2016). Por "valor desconocido" puede entenderse cualquier información sobre un evento que ya ha tenido lugar, que está teniendo lugar en el presente, o que puede tener lugar en el futuro (Provost y Fawcett, 2013). Esta idea es especialmente importante, ya que el término "predictiva" lleva a pensar que esta metodología solo se aplica para realizar estimaciones sobre la probabilidad de que suceda algo en el futuro, cuando también sirve para esclarecer hechos pasados. En la misma línea, la Organización para la Seguridad y la Cooperación en Europa (OSCE, 2017) ha definido la actividad policial basada en la inteligencia como la: "recopilación y evaluación sistemática de datos e información, a través de un proceso analítico definido que los convierte en productos analíticos estratégicos y operativos que sirven de base para un proceso decisorio mejorado, fundamentado y documentado".

La policía predictiva supone un cambio de paradigma en la forma de actuar de los cuerpos policiales, ya que ha permitido pasar de un enfoque reactivo a un enfoque preventivo. Este enfoque proactivo y prospectivo facilita la prevención y reducción de la delincuencia, optimizando los limitados recursos humanos y materiales policiales, puesto que los resultados de este tipo de análisis ayudarán a tomar decisiones tácticas u operativas. Tan importante como desarrollar herramientas $y$ algoritmos predictivos es conseguir aplicar con éxito los resultados de los análisis en la práctica. La mayoría de los trabajos y algoritmos se ocupan de la predicción de futuros crímenes, o del lugar y momento en el que con mayor probabilidad tendrán lugar, pudiéndose distinguir distintas aplicaciones (Perry et al., 2013):

1. Predecir la probabilidad que hay de que tenga lugar un crimen, en un lugar y momento determinados.

2. Predecir la probabilidad que tiene un individuo concreto de cometer un crimen.

3. Predecir el riesgo que tiene un individuo de convertirse en víctima.

4. Predecir las características que, con mayor probabilidad, tendrá el autor de un crimen que ya se ha cometido.

La policía predictiva opera a partir de semejanzas y analogías, es decir que se basa en la identificación de patrones (Kaufman et al., 2019). Un patrón es un conjunto de variables, y las relaciones entre estas, que aparece de forma constante y puede ser identificado dentro de un conjunto de datos mayor (Waske y Beneditksson, 2014). Los estudios sobre el crimen han mostrado de forma consistente que pueden identificarse regularidades en la forma de operar de los criminales en diferentes delitos. Así, por ejemplo, desde la psicología y la sociología ambiental, se ha demostrado que los criminales tienden a actuar en lugares que conocen (zona de confort) y no realizan grandes desplazamientos. Por otro lado, se ha demostrado que la distribución de los crímenes no es homogénea, sino que tiende a concentrarse en lugares y momentos determinados en lo que se conoce como hot spots (Eck et al., 2005).

Los patrones se hacen reconocibles cuando se analizan una gran cantidad de casos, ya que de esta forma se reconocerán aquellas características que se mantienen estables, permitiendo diferenciarlas del resto de información que será algo situacional y puntual. También será necesario recoger cuantas más variables mejor, ya que de esta forma se podrá estudiar la capacidad predictiva de un mayor número de indicadores. Si el conjunto de datos que se analiza es pequeño no será posible reconocer un patrón (Kaufman et al., 2019), e incluso pueden obtenerse conclusiones erróneas, por lo que es necesario trabajar con el mayor número de casos posibles, buscando que esa cantidad sea una muestra representativa del fenómeno que se está analizando. Estos datos, en la mayoría de los casos, solo pueden ser recopilados por los agentes policiales encargados 
de las investigaciones, por lo que desde el ámbito académico es imprescindible facilitar esta tarea, desarrollando estudios que ayuden a identificar las variables clave que se deben recoger. Por esto también es importante la colaboración con otros profesionales, por ejemplo, de la salud o de servicios sociales, que aporten información a la que los agentes policiales no pueden acceder, o que sería muy costoso para ellos. Es por tanto importante destacar la necesidad de compartir, conectar y analizar de forma centralizada, los datos y la información pertinentes obtenidos a todos los niveles, no solo policial, y siempre de conformidad con las leyes nacionales y las normas internacionales en materia de derechos humanos (OSCE, 2017; Ratcliffe, 2016).

Los algoritmos se diseñan para ser aplicados en un tipo de delito concreto, en un lugar concreto, o en un grupo de individuos concreto. Esto quiere decir que los algoritmos no son extrapolables a supuestos diferentes para los que han sido diseñados. Algunos autores (Kaufman et al., 2019; Kitchin, 2014) señalan que la policía predictiva sólo puede aplicarse en delitos que siguen un patrón, por lo que delitos que sean situacionales, o que tengan una naturaleza impulsiva, no podrán predecirse con un grado significativo de precisión. Por lo tanto, parece que existen determinados delitos sobre los que estas técnicas pueden ser aplicadas con mayor eficacia. El problema es que los delitos que mayor alarma social generan son los delitos violentos contra las personas, como los homicidios (especialmente los que se dan en el ámbito de la pareja) o las agresiones sexuales (especialmente aquellas que son cometidas por más de un autor), que presentan un grado de planificación bajo y dependen, en parte, de factores situacionales que no forman parte de un patrón. Además, estos delitos suelen presentar una prevalencia muy baja si se compara con otro tipo de delitos. Por ejemplo, en España, según el Portal Estadístico de Criminalidad del Ministerio del Interior, en el año 2018 se registraron 290 casos de homicidio consumado y 1700 agresiones sexuales con penetración, frente a 302043 delitos de robo con fuerza en las cosas. Pese a estas diferencias, sigue siendo prioritario desarrollar estrategias eficaces para le prevención de aquellos delitos que afectan directamente a las personas.

\section{Problemas derivados de la aplicación de modelos predictivos}

El desarrollo de la policía predictiva ha permitido que, de forma sistemática, se puedan gestionar y analizar datos sobre el crimen con una finalidad práctica. Si bien muchos estudios sobre el crimen se basan en hacer descripciones sobre un fenómeno criminal concreto, o proponen teorías que ayudan a explicar por qué tienen lugar diferentes hechos delictivos, la policía predictiva ha permitido que las ciencias criminalistas (González, 2015) trabajen priorizando la importancia de las implicaciones operativas de los estudios sobre el crimen. Pese a esto, algunos autores han destacado algunos problemas potenciales que pueden derivarse de la aplicación de la policía predictiva.

Uno de los principales problemas es su falta de transparencia (Bland, 2020; Datta et al., 2016; Schlehahn et al., 2015; Strikwerda, 2020). Algunos de los algoritmos que se emplean en la predicción de crímenes funcionan siguiendo un modelo de caja negra, es decir que el usuario tras introducir los datos en el modelo obtiene unos resultados, pero no sabe muy bien cómo se ha llegado a esos resultados. Esto puede dar lugar a dos situaciones. En la primera, el agente policial, al no entender los resultados o el proceso que sigue el algoritmo, no tendrá en cuenta las recomendaciones que le ofrece el sistema, y dará mayor importancia a su experiencia personal (Macnish et al., 2020). En la segunda situación, el agente policial puede entender que los resultados aportados por el algoritmo son suficientes para la toma de decisiones, y no se detendrá a interpretar o dar sentido a los resultados aportados por el sistema. En la primera situación se estarían malgastando recursos en desarrollar herramientas que no son usadas por los agentes, y en la segunda se estaría reduciendo el proceso de toma de decisiones a lo que dice el algoritmo. Ambas situaciones deben evitarse, ya que afectarán negativamente a la eficacia y eficiencia de las actuaciones policiales (Perry et al., 2013). Los investigadores policiales usuarios de estos sistemas deben tener un conocimiento previo del fenómeno que se está analizando, que les sirva para dar una explicación a los resultados de los análisis, y dotarlos de un contexto, lo que les llevará a confiar en esas herramientas y a crear estrategias más eficaces en la lucha contra el crimen (Bennett Mosses y Chan, 2016; Townsley, 2017).

Otro de los problemas se deriva del hecho de que estas herramientas se basan en delitos conocidos y esclarecidos por los departamentos policiales. Como señala Joh (2014), puede haber determinados delitos con una mayor probabilidad de ser investigados y esclarecidos, porque son cometidos por individuos que se consideran peligrosos, o porque tienen lugar en zonas propensas a la delincuencia. $\mathrm{Si}$ los datos que se introducen en el modelo presentan algún tipo de sesgo, se podría provocar la estigmatización de individuos o zonas que presenten las características que los algoritmos identifican como indicadores de riesgo (Strikwerda, 2020). La elaboración de perfiles étnicos, por ejemplo, aparte de ser una práctica discriminatoria contraria a las leyes, ha demostrado su ineficacia (OSCE, 2017). Además, determinadas tipologías delictivas presentan un 
elevado número de casos que no llegan a conocimiento de los investigadores policiales, por lo que no formarán parte de los conjuntos de datos que se empleen en la creación de los algoritmos, generando una visión distorsionada del fenómeno, fundamentada no en el delito en cuestión, sino en los casos que llegan a conocimiento de las autoridades.

Estos problemas basados en la falta de transparencia y la posible estigmatización que pueden perpetuar los algoritmos si se trabaja con datos sesgados, han hecho que varios autores llamen la atención sobre las implicaciones éticas derivadas del uso de estas herramientas, y cómo podrían vulnerar el derecho a la intimidad y las leyes de protección de datos (Ferguson, 2011; OSCE, 2017; Oswald et al., 2018). Al ser uno de los objetivos el predecir futuros delitos, o estimar la peligrosidad futura de un individuo, los detractores de estas herramientas se plantean si es ético actuar de alguna forma contra alguien que aún no ha cometido ningún delito, o si estaría justificado monitorizar las acciones de determinados individuos para estimar su peligrosidad. Por su parte, los defensores de este tipo de prácticas policiales, argumentan que estas herramientas ayudan a reducir los sesgos de los investigadores, ya que sustituyen la experiencia personal por un conocimiento basado en el análisis sistemático de todos los casos conocidos y esclarecidos (Ferguson, 2015; Koss, 2015).

Respondiendo a algunas de las críticas que reciben los modelos de policía predictiva, cabe destacar que el desarrollo de estas prácticas es la evolución lógica de la práctica policial tradicional. Dada la incorporación de las nuevas tecnologías en todos los ámbitos de la sociedad, es coherente pensar que también sean aplicadas en el análisis del crimen. Hasta ahora, los departamentos de policía ya recogían de forma sistemática información sobre delitos conocidos, conformando las bases de datos policiales. $\mathrm{Si}$ bien estos repositorios permitían realizar consultas puntuales, las técnicas de policía predictiva generan conocimiento útil, que puede ser aplicado en las investigaciones de nuevos hechos delictivos, y puede ayudar a gestionar de una forma más eficiente los recursos policiales que, en muchos casos, son limitados.

\section{La policía predictiva y su aplicación en España}

En suma, el desarrollo de las técnicas de investigación cuantitativas permitirá a los cuerpos policiales mejorar la gestión de recursos, haciéndolos más eficaces y eficientes en la lucha contra determinados delitos. En este sentido, Liberatore et al. (2018) destacaron como principales ámbitos de aplicación de la policía predictiva en España los siguientes:
1. Predicción de crímenes.

2. Delimitación de distritos policiales y optimización de zonas de patrullas.

3. Análisis y vinculación de crímenes en serie.

4. La estimación del riesgo de nuevos episodios de violencia de género.

5. Análisis de las vulnerabilidades de las redes de delincuencia organizada.

6. Análisis de imágenes de cámaras policiales.

7. Análisis de la productividad laboral.

8. Detección de denuncias falsas.

De hecho, en España la policía predictiva ya se ha aplicado en diferentes tipologías delictivas. Siguiendo un orden cronológico, en 2006, a raíz de una serie de incendios forestales en Galicia, la Guardia Civil impulsó un estudio sobre los perfiles del incendiario forestal (González, 2011; González et al., 2010; Sotoca et al., 2013), en colaboración con la Fiscalía Coordinadora de Medio Ambiente y Urbanismo de la Fiscalía General del Estado. Para ello, se dispuso que los agentes de las unidades policiales encargadas del esclarecimiento de estos delitos, a lo largo de todo el territorio nacional, cumplimentaran online un cuestionario psicosocial cada vez que detuvieran a un incendiario (González et al., 2017), complementario a las diligencias policiales. Con esa información se llegó a programar un sistema predictivo utilizando redes bayesianas (Delgado et al., 2016; 2018), que informa de las características más probables de un incendiario desconocido a partir de los indicios encontrados en el foco del incendio, para ayudar a los agentes a su localización e identificación. Aunque este sistema dista mucho de estar terminado, ha marcado el camino a seguir en otras modalidades delictivas.

El procedimiento policial predictivo más desarrollado en España es el que tiene que ver con la creación y validación del protocolo de valoración policial del riesgo de reincidencia en el ámbito de la Violencia de Género, del Sistema de Seguimiento Integral de los Casos de Violencia de Género (Sistema VioGén; González-Álvarez et al., 2018), desarrollado desde la Secretaría de Estado de Seguridad (SES) del Ministerio del Interior. Este protocolo permite que los agentes valoren el riesgo que tiene una mujer que denuncia, de sufrir una nueva agresión por parte de quienes son o fueron sus parejas varones. Para ello se emplea la herramienta informatizada denominada Valoración Policial del Riesgo (VPR; López-Ossorio et al., 2017). En función del nivel de riesgo resultante, el protocolo contempla la adopción de determinadas medidas de protección policial que pretenden evitar esa reincidencia, gestionándose de este modo el riesgo 
(López-Ossorio et al., 2019; López-Ossorio, Loinaz et al., 2019). Una vez más, esto refleja que, en materia de policía predictiva, las estimaciones deben ir acompañadas de intervenciones de algún tipo. Este protocolo se ha ido mejorando con el tiempo, hasta llegar en la actualidad a la versión 5.0, en la que, como se explicará en detalle más adelante, se incluye una escala de valoración del riesgo de violencia letal (López-Ossorio et al., 2020).

En 2015, la Policía Nacional aplicó modelos predictivos a uno de los problemas clásicos a los que han tenido que enfrentarse los departamentos de policía durante años: la delimitación de zonas de patrullaje (Camacho-Collados y Liberatore, 2015; Camacho-Collados et al., 2015; Mitchell, 1972; Wang, 2012; Liberatore y Camacho-Collados, 2016). El principal reto en este ámbito está en que la delimitación de estas zonas debe estar basada en atributos relevantes (Kalcsics et al., 2005), entre los que estarían las características sociales, las topológicas y la distribución del crimen en un espacio y en un momento concreto. Gracias al desarrollo de los Sistema de Información Geográfica (SIG), ha sido posible gestionar y analizar datos espacio-temporales en un tiempo razonable, lo que ha permitido identificar concentraciones de hechos delictivos. Así, el Sistema de Soporte a la Decisión (SSD) propuesto por Camacho-Collados (2016) para la Policía Nacional, tenía como objetivo principal implementar un patrullaje predictivo que ayudara a distribuir la presencia de agentes policiales en aquellos lugares y momentos con mayor concentración de delitos, buscando reducir la probabilidad de ocurrencia de los mismos. Los experimentos demostraron que la aplicación de este patrullaje predictivo en la ciudad de Madrid podría mejorar los modelos de distribución convencionales. Además, se introdujo el concepto de apoyo mutuo, que permite que se pueda contar con la ayuda de los efectivos de los distritos cercanos, redistribuyendo en tiempo real, si fuera necesario, los recursos disponibles en un lugar y momento determinados (Camacho-Collados y Liberatore, 2015; Camacho-Collados et al., 2015).

Otro problema que afrontó la Policía Nacional fue el de las denuncias falsas. Este fenómeno delictivo tiene un gran coste para los cuerpos policiales, en primer lugar, porque se destinan medios a investigar delitos que realmente no han sido cometidos, impidiendo que estos recursos puedan ser empleados en la investigación de delitos reales. Y, en segundo lugar, porque este tipo de hechos obstaculizan el trabajo policial, siendo además necesario investigar el delito de simulación una vez se descubre la falsedad de la denuncia (Liberatore et al., 2018). Para dar una respuesta inicial a este problema, en 2017 se creó en la Policía Nacional la herramienta informática VeriPol, que permite estimar la probabilidad de que una denuncia por robo sea falsa (Quijano-Sánchez et al., 2018). VeriPol basa su análisis en el procesamiento del texto de la denuncia, lo cual presenta dos importantes ventajas: 1) no es necesario que los investigadores incluyan más datos que el testimonio facilitado por el denunciante; y 2) los investigadores reciben inmediatamente el resultado de la valoración que realiza VeriPol, lo cual permite tomar decisiones más acertadas desde el comienzo de la investigación, evitando malgastar recursos policiales. El estudio piloto llevado a cabo por Liberatore et al. (2018) en las ciudades de Murcia y Málaga, mostró un incremento en el número de casos de falsas denuncias esclarecidos del $839.4 \%$ y $303.6 \%$ respectivamente, presentando la herramienta además un $90 \%$ de acierto. Pero no solo son importantes las cifras sobre el rendimiento de VeriPol, sino cómo se integró en el trabajo policial diario, y la recepción que tuvo entre los investigadores policiales, que son los usuarios finales de este tipo de sistemas. En ese mismo estudio piloto, los policías que pudieron hacer uso de VeriPol consideraron que era una herramienta útil, que sería fácil de implementar en su trabajo diario y, lo más importante, que harían uso de VeriPol, recomendando su implementación en todo el territorio nacional, proponiendo incluso que debería ampliarse a otras tipologías delictivas.

Otro de los ámbitos de aplicación de la policía predictiva es la delincuencia sexual. En España se desarrolló con fines predictivos una investigación centrada en las agresiones sexuales cometidas por autores desconocidos para las víctimas (GiménezSalinas et al., 2018). En la actualidad desde la SES se impulsa un proyecto científico para validar la versión española de una herramienta de priorización policial para casos de online child grooming, denominada Chat Analysis Triage Tool (CATT; Seigfried-Spellar et al., 2019). Su objetivo es identificar, mediante el análisis del discurso y las tácticas utilizadas por los groomers en los chats, a aquellos abusadores que pretenden tener un encuentro físico con el menor, y diferenciarlos de aquellos que solo buscan satisfacer sus fantasías sin buscar un contacto real. Para ello, la herramienta analiza los chats mediante algoritmos que identifican a aquellos investigados que con mayor probabilidad puedan tener un contacto físico con el menor, permitiendo priorizar las actuaciones y recursos policiales sobre estos.

Por su parte, la Secretaría General de Instituciones Penitenciarias ha venido desarrollando iniciativas para detectar y prevenir los procesos de captación y de radicalización de internos, principalmente musulmanes, en los Centros Penitenciarios. La Orden de Servicios 3/2018 
propició la creación de un instrumento de evaluación del riesgo de radicalismo violento, que utiliza regularmente desde su entrada en vigor en el mes de febrero de ese año. El instrumento se elaboró a partir de la revisión de otras herramientas de evaluación del riesgo que existen a nivel internacional (e.g., TRAP18, VERA-2R, ERG 22+ y MLG), realizándose las adaptaciones oportunas al contexto penitenciario español (Fernández, 2019). Dicho instrumento tiene como objetivo general la detección y valoración del riesgo de reincidencia de aquellos internos que han cometido delitos de terrorismo yihadista, y la detección de internos que se encuentran en proceso de radicalización o que son vulnerables a ser captados. Y como objetivos específicos presenta: a) ayudar en la toma de decisiones en lo relativo al tratamiento penitenciario, reorientando la detección, seguimiento e intervención; b) servir de instrumento de coordinación entre los distintos departamentos penitenciarios, en particular, las áreas de seguridad y tratamiento; y c) sistematizar el tratamiento sobre el radicalismo violento llevado a cabo en los distintos Centros Penitenciarios.

Finalmente, en la actualidad, el Centro Nacional de Desaparecidos está trabajando en la elaboración de una herramienta para predecir el tipo de desenlace más probable de la desaparición de personas, especialmente valorando el riesgo de que la persona sea encontrada fallecida por causas violentas. Los agentes policiales podrán hacer uso de la herramienta desde el mismo momento en que se recibe la denuncia, lo que permitirá, por ejemplo, intensificar la búsqueda en lugares significativos para la víctima (en caso de que pueda tratarse de un suicidio), o para activar sin dilación a los equipos de investigación de homicidios y no perder así pruebas valiosas que puedan desparecer con el paso del tiempo (García-Barceló et al., 2020; García-Barceló et al., 2019).

Para ejemplificar los desarrollos y aplicaciones de la policía predictiva en España, en los siguientes apartados se va a profundizar en dos de ellos. Primero, en la estimación del riesgo de reincidencia de los casos denunciados por Violencia de Género y la gestión de la seguridad de las víctimas, que se realizan a través del Sistema VioGén, que ha evolucionado con los años hasta la creación del formulario $\mathrm{VPR}_{5.0}-\mathrm{H}$. Y, en segundo lugar, los inicios del desarrollo de una herramienta que permita a los investigadores policiales, ante un nuevo caso de homicidio, hacer una estimación probabilística de las características que tendrá el autor.

\section{Policía predictiva y Violencia de Género en España. La predicción del feminicidio}

El 30\% de las mujeres que han tenido pareja han sufrido violencia física o sexual por parte de esta (Organización Mundial de la Salud [OMS], 2013). Pese a la elevada prevalencia que tiene este fenómeno en todo el mundo, los estudios señalan que tan solo una parte de la violencia que sufren las mujeres en las relaciones de pareja se denuncia (Gracia, 2004; Narayan et al., 2019). Además, la preocupación por estos casos se ve incrementada por la existencia de delitos especialmente violentos perpetrados por la pareja de la víctima, como serían las agresiones sexuales (Stockman et al., 2013) o el homicidio de pareja, también denominado feminicidio (Stöckl et al., 2013). En el caso español, este tipo de violencia está regulada por la Ley Orgánica 1/2004 de Medidas de Protección Integral contra la Violencia de Género. La Macroencuesta de Violencia Contra la Mujer (2020) encontró que el $32.4 \%$ de las mujeres mayores de 16 años en España había sufrido algún tipo de violencia por parte de su pareja actual o expareja. También se encontró que sólo el $21.7 \%$ de las mujeres que han sufrido este tipo de violencia denunciaron, reduciéndose este porcentaje al $5.4 \%$ cuando es la pareja actual la que ejerció esa violencia.

El elevado número de denuncias, y sobre todo la preocupación social, han hecho que la lucha contra este delito sea un objetivo prioritario para las fuerzas policiales de todo el mundo, tornándose prioritaria la gestión de los recursos policiales en este ámbito. Por esto precisamente es por lo que se ha impulsado la creación de algoritmos y herramientas centrados en la predicción y gestión del riesgo de los casos de violencia de pareja (Bland, 2020), existiendo a nivel mundial diferentes herramientas (e.g., Dayan et al., 2013; Hiltonet al., 2008; Kropp y Hart, 2000), $y$, entre ellas, dos que tienen como objetivo la predicción de la violencia mortal, el Danger Assessment (Campbell, 2012) y la VPR $5.0-\mathrm{H}$ española (López-Ossorio et al., 2020).

\section{Homicidio y feminicidio}

El homicidio intencionado consiste en terminar con la vida de otra persona de manera voluntaria o dolosa, y es considerado como la manifestación más extrema de la violencia y del comportamiento criminal (González et al., 2018). El derecho a la vida es uno de los derechos humanos fundamentales, que en España se recoge en el artículo 15 de la Constitución, y su protección es uno de los principales objetivos de cualquier Estado moderno. No en vano, el número de muertes violentas se emplea como indicador del nivel de seguridad y de desarrollo de un país. Además, en las sociedades más desarrolladas, el homicidio preocupa especialmente cuando se produce en el ámbito doméstico, sobre todo en las relaciones de pareja, porque en este entorno se supone que es donde mayor protección y 
seguridad debe existir. En este sentido, la proporción de mujeres víctimas de este tipo de homicidios es hasta seis veces mayor que para el caso de los hombres (Stöckl et al., 2013), por lo que estos crímenes se vinculan al fenómeno general de violencia contra la mujer, y que es definida internacionalmente como aquella violencia que se ejerce contra la mujer por el hecho de serlo.

Aunque España presenta una tasa de homicidios inferior a la tasa europea y mundial, sucediendo esto también en el caso concreto de los homicidios de pareja (2.81 frente al 3.94 y 5.04 por millón de mujeres mayores de 14 años, respectivamente), cuenta con políticas activas para combatir y reducir la Violencia de Género (Ley Orgánica 1/2004 de Medidas de Protección Integral contra la Violencia de Género). Pese a esto, se registra una media anual de 60 feminicidios, lo que supone aproximadamente una cuarta parte de las muertes violentas en España, y más de la mitad de los casos de mujeres que mueren de forma violenta en España.

\section{Equipo nacional de revisión de feminicidios}

Si la sociedad se plantea el objetivo de erradicar estas muertes, lo primero que se debe hacer es conocer el fenómeno y estudiarlo con atención, para aislar los indicadores de riesgo que permitan anticipar o predecir específicamente el feminicidio. Tras una revisión de la literatura (López-Ossorio et al., 2018), en España se abordó esta cuestión creándose el Equipo Nacional de Revisión Pormenorizada de Homicidios en el contexto de la Violencia de Género en España (EHVdG; González, Garrido, et al., 2018), en el que se implicaron todas las Instituciones gubernamentales con responsabilidades en esta materia, y entre las que están: la Secretaria de Estado de Seguridad del Ministerio del Interior, la Fiscalía coordinadora contra la violencia sobre la mujer, la Delegación del Gobierno para la violencia de género y el Observatorio contra la violencia doméstica y de género del Consejo General del Poder Judicial.

La prevalencia del fenómeno en España obligaría a revisar casos de diferentes años y de todo el territorio nacional, para conseguir una muestra lo suficientemente grande y representativa tanto a nivel estadístico (estimada en unos 200 casos) como a nivel territorial. En los informes documentales (e. g., atestados policiales, sentencias judiciales, informes penitenciarios o informes médicos) de cada caso no se suelen recoger datos psicosociales ni de la víctima mortal ni del agresor, ni sobre su dinámica relacional en las fechas próximas al feminicidio, por lo que para llegar al nivel explicativo y superar el mero análisis descriptivo sería necesario localizar y entrevistar a personas próximas a ambos implicados, con los costes pertinentes en cuanto a recursos materiales y humanos se refiere. Para realizar esta tarea en un plazo temporal razonable habría que contar con una cantidad suficiente de entrevistadores, que trabajaran en grupo (para asegurar la fiabilidad de los datos), y con preparación en materia de entrevista de investigación, perfilamiento indirecto y autopsia psicológica. Esas entrevistas deberían seguir un guion de entrevista semiestructuradas (protocolo estandarizado). Por último, sería de vital importancia poder acceder a realizar una entrevista voluntaria en prisión con los autores, en los casos en los que estos no se hayan suicidado.

Tomando en cuenta todas estas circunstancias, desde la SES se asumió la tarea de coordinar este ambicioso estudio, congregando a nivel central a expertos de las diferentes instituciones implicadas, así como de las Fuerzas y Cuerpos de Seguridad, Secretaría General de Instituciones Penitenciarias y Colegio Oficial de Psicólogos de Madrid. Este equipo central revisó la bibliografía al respecto del feminicidio a nivel internacional y las buenas prácticas de equipos homólogos de otros países. También se ocupó de planificar e impulsar la revisión de los casos en España, facilitando para ello la organización de grupos de revisión provinciales, formados por expertos de más de 20 universidades e instituciones de investigación científica: profesores y alumnos de posgrados, especialmente psicólogos forenses y criminólogos, que, en virtud de convenios de colaboración con el Ministerio del Interior, acometieron los trabajos de revisión de campo, previa capacitación a cargo de la coordinación del equipo nacional. De este modo se armó un proyecto de investigación científica que ha resultado pionero en España, tanto por la cantidad y variedad de instituciones implicadas, como por la dimensión de la tarea.

El personal investigador (formado finalmente por más de 450 integrantes) asumió la responsabilidad de revisar de forma pormenorizada sobre el terreno los feminicidios, asumiendo los siguientes cometidos:

1. Estudio del material documental policial (atestados, diligencias e inspecciones técnico oculares), judicial (sumarios e informes periciales) y penitenciario.

2. Identificación y contacto con posibles informantes.

3. Desplazamiento hasta los lugares en donde ocurrieron los hechos.

4. Entrevista semiestructurada y habilidosa a implicados relacionados con la víctima y el autor (para recopilar datos 
sociodemográficos y criminológicos inexistentes en el material documental).

5. Entrevista en profundidad con el propio autor en el establecimiento penitenciario (con carácter voluntario).

6. Análisis de los datos recopilados.

7. Emisión del correspondiente informe, en el que se recopilan los hallazgos respecto a los indicadores de riesgo presentes en cada caso y circunstancias que facilitaron, o impidieron, su adecuada identificación en el momento del crimen, especialmente la coordinación institucional.

Para compensar los costes de la revisión pormenorizada, el Ministerio del Interior concedió cinco proyectos de investigación, en distintos territorios, a las universidades de Santiago de Compostela, Valencia, Granada, Málaga, y a la Fundación Universitaria Behavior \& Law; y desde la Comunidad Madrid se concedió uno a la Universidad Autónoma de Madrid. Fruto de todos esos esfuerzos, se consiguió revisar satisfactoriamente 171 feminicidios, habiéndose descartado 30 casos por razones que impidieron completar la tarea de revisión.

\section{$\mathrm{VPR}_{5.0}-\mathrm{H}$. La valoración del riesgo de violencia mortal}

Uno de los resultados preliminares más relevantes del estudio de revisión pormenorizada de feminicidios, ha sido la construcción y validación de la escala de predicción del riesgo de resultado letal cuando se formula una denuncia por Violencia de Género en España. Para la creación de la escala, denominada $\mathrm{H}$, se siguió un diseño de casos y controles, estando la muestra final compuesta por 2159 casos. De estos, 159 (7.4\%) fueron feminicidios y $2000(92.6 \%)$ casos no mortales que actuaron de grupo control. Para llevar a cabo el estudio, fueron necesarias las VPR de todos los casos, ya que el objetivo era saber si los indicadores que predicen reincidencia violenta serían capaces de predecir también un episodio mortal. Según la Delegación del Gobierno contra la Violencia de Género, de los 60 feminicidios de media que se vienen registrando en España entre los años 2006 a 2019, solo en un 26\% se contaba con denuncia previa. Esto puede deberse a dos motivos: 1) que la víctima no denuncia episodios de violencia anteriores; o 2) que no existían episodios violentos previos. Para solventar este problema, y poder contar con la VPR de los 159 incluidos en el estudio, fue determinante la tarea del EHVdG, ya que las VPR de aquellos feminicidios que no contaban con denuncia previa fueron cumplimentadas por los equipos de revisión. Los casos del grupo control se seleccionaron de forma aleatoria de entre aquellos registrados durante el último cuatrimestre de 2016 en el Sistema VioGén.

De este modo, se pudo llegar a identificar un subconjunto de 13 indicadores que se emplearon para la construcción de la herramienta de predicción de violencia mortal, y que presentó una sensibilidad de .81, una especificidad de .61 y un área bajo la curva (AUC) de .81 [.76, .86]; mostrando además un valor predictivo positivo $(\mathrm{VPP})=.19$, $\mathrm{y}$ un valor predictivo negativo (VPN) =.97 (López-Ossorio et al., 2020). Se encontró que los pesos de cada uno de los indicadores de la VPR utilizados para construir el algoritmo de predicción de la reincidencia, no servían para predecir adecuadamente los feminicidios, y viceversa. Este hecho es, en cierto modo, esperable, ya que la bibliografía científica ha mostrado de forma consistente que violencia mortal y no mortal en la pareja pueden ser fenómenos diferentes, y por lo tanto, pueden asociarse a circunstancias diferentes. Esta escala se incluyó en la herramienta de predicción de riesgo, de tal forma que en el formulario (desde marzo de 2019 el VPR 5.0 ) se incluyen dos escalas con algoritmos diferentes: una para estimar los riesgos de reincidencia con cinco niveles (no apreciado, bajo, medio, alto y extremo), y otra para estimar el riesgo de feminicidio con dos niveles (en bajo y alto).

Con el objetivo de facilitar a los miembros de las fuerzas y cuerpos de seguridad sus decisiones en materia de protección de las víctimas, con esta evidencia se optó por programar un mecanismo dual y transparente para los agentes, del siguiente modo: cuando se recibe la denuncia de un caso, los policías cumplimentan la VPR. En este momento, sin mostrar todavía el resultado, el Sistema VioGén aplica el primer algoritmo y calcula el riesgo de reincidencia que presenta el caso en ese momento, e inmediatamente después, calcula el riesgo de feminicidio con el segundo algoritmo. En caso de que aparezca riesgo mortal, se ha dispuesto que se incremente en un nivel el riesgo de reincidencia, que es el que se muestra finalmente a los agentes, junto con una alerta de que el caso es de especial interés, para que se pueda adecuar la protección policial a las características del caso concreto. Además, esta alerta se deja reflejada en una diligencia en el atestado policial, que se envía al Juzgado y a la Fiscalía competentes, para conocimiento de la singularidad del caso, y por si estimaran pertinente que los implicados fueran evaluados cuanto antes por psicólogos o médicos forenses, quienes podrían profundizar más en las circunstancias del caso y proponer nuevas medidas protectoras. Con este mecanismo dual se espera reducir la tasa de feminicidios en los casos que se denuncien en el territorio en donde se utiliza el Sistema VioGén. Al haberse puesto en explotación en la primavera de 
2019, y tratarse de un fenómeno criminal con baja prevalencia, habrá que esperar varios años para conocer la eficacia real de este protocolo dual, estudiando la evolución de los casos alertados.

\section{Lecciones aprendidas}

El Sistema VioGén, la VPR ${ }_{5.0}-\mathrm{H}$, y en general el fenómeno de la violencia de pareja en España, permiten ilustrar de forma clara algunos de los problemas que pueden presentarse en el desarrollo de las aplicaciones de policía predictiva; pero también los beneficios que tiene, y sobre todo cuales son algunos de los retos futuros que se deben afrontar. En primer lugar, se ha visto que para llegar a acumular suficiente información se tuvo que hacer un gran esfuerzo por no encontrarse en repositorios informatizados y centralizados. Esta necesidad de recopilar adecuadamente, compartir y analizar centralizadamente los datos relevantes, es clave en materia de policía predictiva (OSCE, 2017).

Por otra parte, el hallazgo de que no todos los indicadores de la VPR que son útiles para la predicción de reincidencia, lo son para predecir futuros episodios mortales, y el hecho de que, además, fuera necesario recalcular los pesos de los 13 indicadores significativos, es indicativo, como ya han mostrado otros trabajos previos (Matias et al., 2019; Spencer y Stith, 2018), de que violencia mortal y no mortal pueden ser fenómenos diferentes, aunque ambas se den en el marco de la Violencia de Género. Desde el punto de vista de la aplicación de la Inteligencia Artificial (IA) a la predicción del crimen, se plantea una importante reflexión, y es ¿qué nivel de análisis es necesario entonces para desarrollar algoritmos que realmente sean útiles? Normalmente, cuando se hace uso del término Violencia de Género o violencia de pareja, este puede referirse a una variedad de comportamientos violentos que van desde un insulto hasta una agresión mortal, pasando por la existencia de amenazas, o la comisión de una agresión sexual.

En España preocupan especialmente los casos de mujeres que mueren a manos de sus parejas, y que previamente habían denunciado malos tratos (Santos y González, 2017), ya que son entendidos como casos en los que las medidas de protección han fallado, destacando sobre todo cuando el nivel de riesgo de los casos era "bajo" o "no apreciado". Pero hay que entender que, hasta su penúltima versión, la $\mathrm{VPR}_{4.0}$ estaba orientada a la predicción de violencia no mortal, por lo que se podían dar casos, aparentemente paradójicos, en los que mujeres con un riesgo bajo de reincidencia acabaran siendo asesinadas. Los hallazgos más recientes han evidenciado que tal paradoja no existe, y que el problema reside en exigir ingenuamente resultados a una herramienta para predecir un fenómeno para el que no ha sido diseñada.

Por último, es importante destacar que las técnicas de policía predictiva se basan en el análisis de datos históricos, es decir, casos que llegan a conocimiento de los cuerpos policiales. Es por esto que los algoritmos que se generen serán específicos para esos casos, y permitirán predecir casos que muestren características similares o sigan un mismo patrón. El problema, como ya se ha señalado, es que tanto en la Violencia de Género, como en la violencia doméstica en general, muchos casos no llegan a denunciarse, y en consecuencia no forman parte de los registros policiales históricos. Esto plantea otra reflexión importante, y es que, con estos casos no denunciados pueden suceder dos cosas: 1) que sean similares a los casos que sí denuncian, por lo que las herramientas podrían ser aplicables; o 2) puede que tengan una serie de características distintivas que, en parte, expliquen el por qué no se denuncia, y las herramientas de predicción no sirvan. De cualquier modo, lo importante es fomentar que las víctimas denuncien, no solo porque con ello se les podrán aplicar medidas de protección, sino porque cuanto menor sea el número de casos conocidos, más preciso será el conocimiento que se tenga sobre el fenómeno, lo que permitirá diseñar herramientas de predicción más precisas, y que puedan aplicarse en todo tipo de casos, lo que revertirá en último término en mejoras en la protección de las víctimas.

\section{El uso de los datos en la investigación del homicidio en España}

En un trabajo previo realizado en España con datos de la Guardia Civil, Santos et al. (2019) ya destacaron la utilidad de crear bases de datos con información de homicidios esclarecidos, y cómo su análisis podía ayudar en la investigación de nuevos casos de homicidio. El objetivo de aplicar en estos crímenes la metodología de policía predictiva, es el de estimar las características que, con mayor probabilidad, tendrá el autor, o autores, de un nuevo homicidio. Este tipo de razonamiento se basa en la hipótesis de la homología propuesta por Canter (2004), y que viene a expresar que, partiendo del axioma de que la forma en que un criminal comete un delito reflejará las características de este, se puede entender que crímenes con características similares serán cometidos por criminales que compartan determinadas características. La aplicación de esta metodología inferencial inductiva es la base de la policía predictiva (Perry et al., 2013), y es que, como ya señalaron Provost y Fawcett (2013), la policía predictiva no solo se ocupa de estimar lo que puede pasar en el futuro, sino que también puede ayudar a esclarecer algo que ya ha pasado. 
Pese a que aquí no se pretende identificar zonas o momentos de mayor riesgo, predecir una conducta delictiva futura, o estimar el riesgo de que una persona concreta vaya a cometer un acto violento, la finalidad sigue siendo reconocer patrones y aportar información útil a los investigadores policiales. En este caso los patrones son la relación que existe entre un tipo determinado de criminal y las características del crimen. De esta forma, ante un nuevo caso, si se dispone de suficiente información de cómo se cometió el crimen, pueden hacerse estimaciones probabilísticas de las características del agresor. Para identificar estos patrones se ha empleado el software Graphext que permite crear redes (disposición de casos), a partir de la similitud entre nodos (casos) con un concepto de distancia euclídea en tantas dimensiones como variables se registren en el conjunto de datos. Para calcular las dimensiones de las variables numéricas se emplea la distancia de Mahalanobis, y para las dimensiones de las variables categóricas el TD-IDF. Una vez calculadas las medidas, se identifican nodos que comparten características y se genera la disposición final de la red aplicando el algoritmo foceAtlas2; finalmente se aplica sobre la red resultante el algoritmo Louvain, que identifica los clústeres (comunidades) asignándoles un color.

En la Figura 1 se representan todos los casos incluidos en el estudio sobre el Homicidio en España 2010-2012 (González et al., 2018). El concepto de caso hace referencia a la interacción concreta entre las características de la víctima, el autor y las circunstancias del hecho. Una de las ventajas de Graphext es que permite identificar de manera visual los casos similares entre sí, tanto por la proximidad entre los mismos, como por el código cromático. Aunque la clasificación de los casos se genera automáticamente, corresponde al investigador nombrar a los diferentes grupos encontrados en función de aquellas variables que los distinguen del resto.

Figura 1. Distribución de los 871 casos incluidos en el estudio sobre el Homicidio en España 2010-2012.

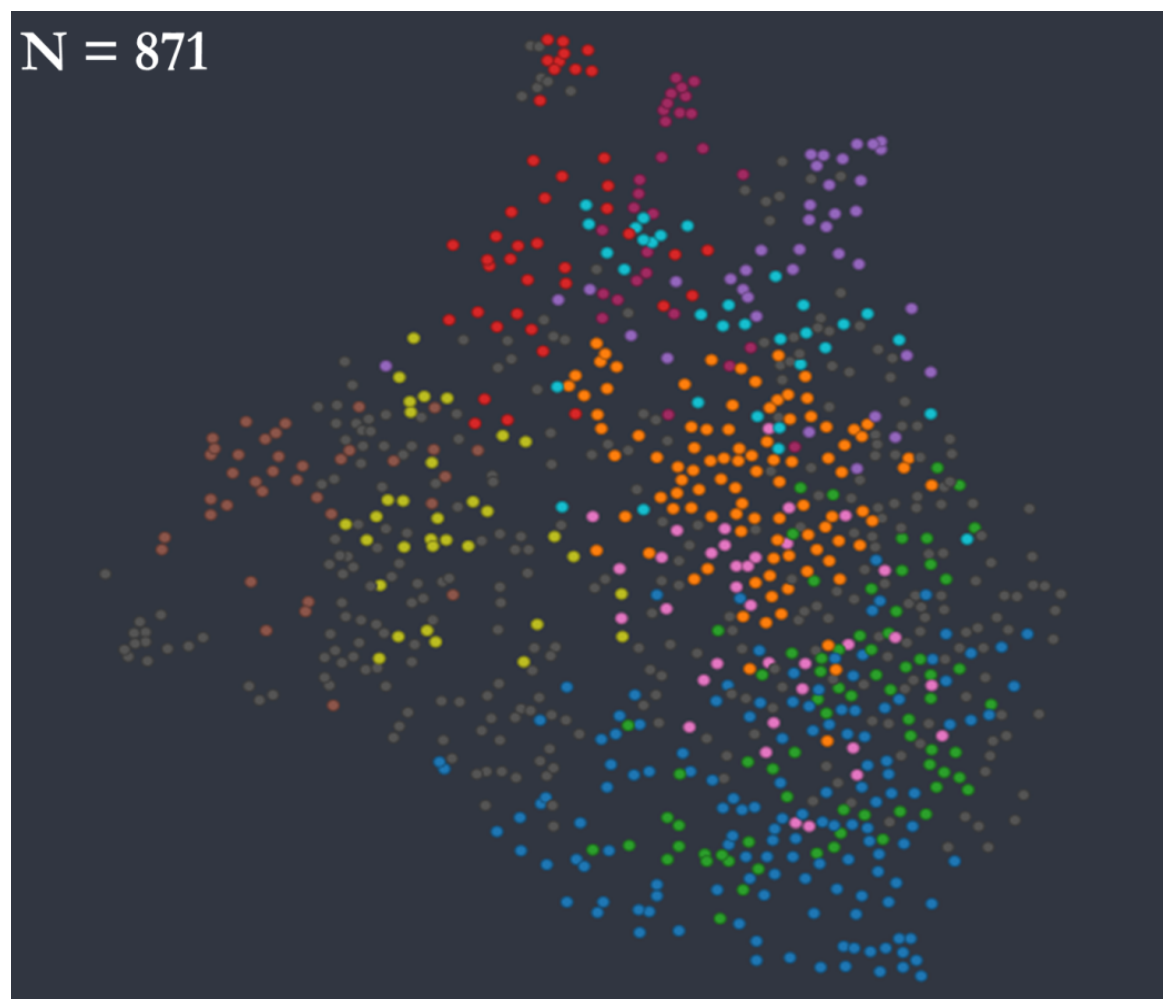

Para ver qué caracteriza a cada tipo de homicidio, Graphext permite seleccionar de forma individual cada una de las variables que componen la base de datos, y asignar un color a cada una de las categorías de esas variables. Como se puede ver en la Figura 2, en el gráfico de la izquierda se ha seleccionado la variable de relación. Su distribución muestra que los casos en los que la víctima tenía una relación más estrecha con el autor se sitúan en la parte inferior de la red (verde $=$ pareja/expareja; rojo $=$ familiar) y a medida que se sube en la red el grado de relación va disminuyendo (azul $=$ conocidos; naranja $=$ desconocidos). Otra de las variables importantes que permite caracterizar un homicidio es si este se comete en el marco de otra actividad criminal. En el gráfico de la derecha se ha seleccionado el tipo de homicidio, encontrándose una concentración de casos relacionados con actividades criminales (naranja) en la parte izquierda de la red. 
Figura 2. Distribución de variables clave.

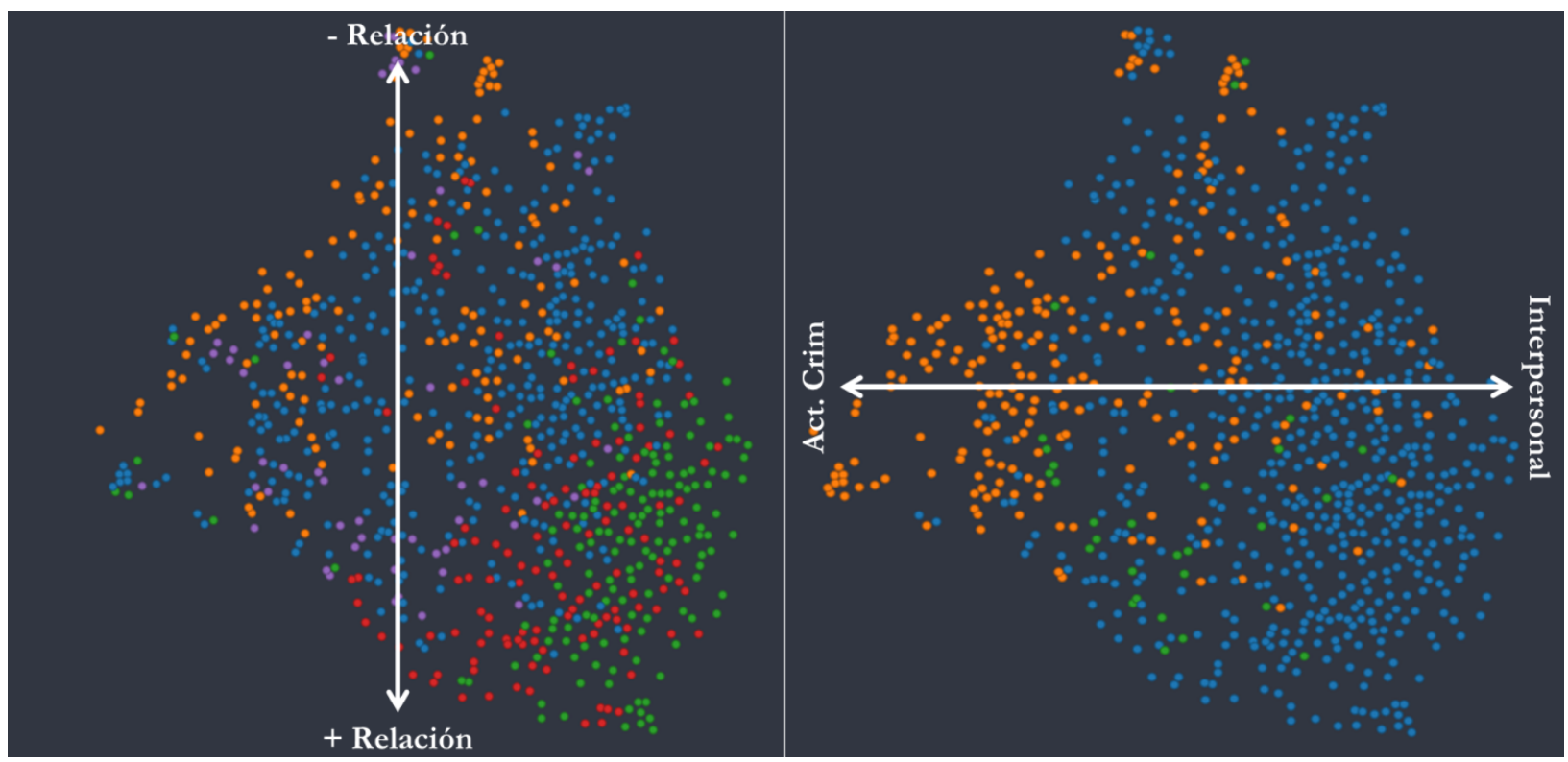

De esta forma se podrían analizar todas las variables que componen la base, y ver su distribución específica. Para facilitar esta tarea, Graphext permite identificar cuáles son las variables que mejor diferencian los clústeres. Una vez identificadas las distribuciones de todas las variables clave, se puede proceder a nombrar cada uno de los grupos que se identifican en la red. Para ilustrar esto, en la Figura 3 se ha asignado un color a cada tipo de homicidio identificado en base a la tipología propuesta por González et al. (2018) para los homicidios en España. Como se puede ver, abajo a la derecha están los casos de Violencia de Género y familiares, que son casos en los que hay una fuerte relación entre víctima y autor. Por otro lado, a la izquierda de la red se puede identificar la concentración de homicidios relacionados con otras actividades criminales. Pese a la clasificación que se puede hacer de los homicidios, en ocasiones pueden registrarse casos con el mismo color pero que estén alejados entre sí, e incluso que estén muy cerca de casos con otro color. La razón de esto es que, aunque los casos pertenecen a un grupo concreto, pueden compartir características con otros homicidios. Esto se ve muy claro en el caso de los homicidios de Violencia de Género y de familiares que en ocasiones están muy juntos entre sí, y lo mismo con los diferentes tipos de homicidios relacionados con actividades criminales.

Hasta ahora, se ha mostrado la capacidad para identificar características que distinguen entre diferentes casos de homicidio, y cómo estos se pueden clasificar. Pero ¿cómo pueden aplicarse estos datos y análisis a la investigación de nuevos hechos delictivos? Como se comentó al comienzo del apartado, la finalidad de identificar características y agrupaciones es conseguir vincular determinadas características del homicidio a un tipo de agresor concreto. Una vez hecho esto, cuando se presenta un nuevo caso de homicidio es necesario recoger la mayor cantidad de información posible. Esta información servirá de filtro para encontrar casos con similares características en los registros históricos de homicidios esclarecidos. Así por ejemplo, si se sabe que la víctima es un varón menor de edad, estas características se introducen en Graphext y se resaltan los casos coincidentes. Como se muestra en la Figura 4, con esas características se han encontrado 44 coincidencias que parece que se agrupan en dos tipos de homicidios claramente diferenciados. Si los investigadores policiales pudieran tener acceso a este tipo de información desde el primer momento en el que se tiene constancia del homicidio, ya podrían plantear dos líneas de investigación, una relacionada con el mundo delincuencial (puntos granates); y otra asociada a posibles conflictos con su entorno familiar (puntos naranjas). El tiempo es muy importante especialmente en las investigaciones policiales de los delitos contra las personas, porque si no se identifican correctamente las diferentes líneas de investigación, existe el riesgo de que los indicios se degraden o que los propios autores los destruyan. 
González-Álvarez, J. L., Santos-Hermoso, J. \& Camacho-Collados (2020). Policía predictiva en España. Aplicación y retos de futuro. Behavior \& Law Journal, 6(1), 26-41.

Figura 3. Identificación de tipos de homicidio.

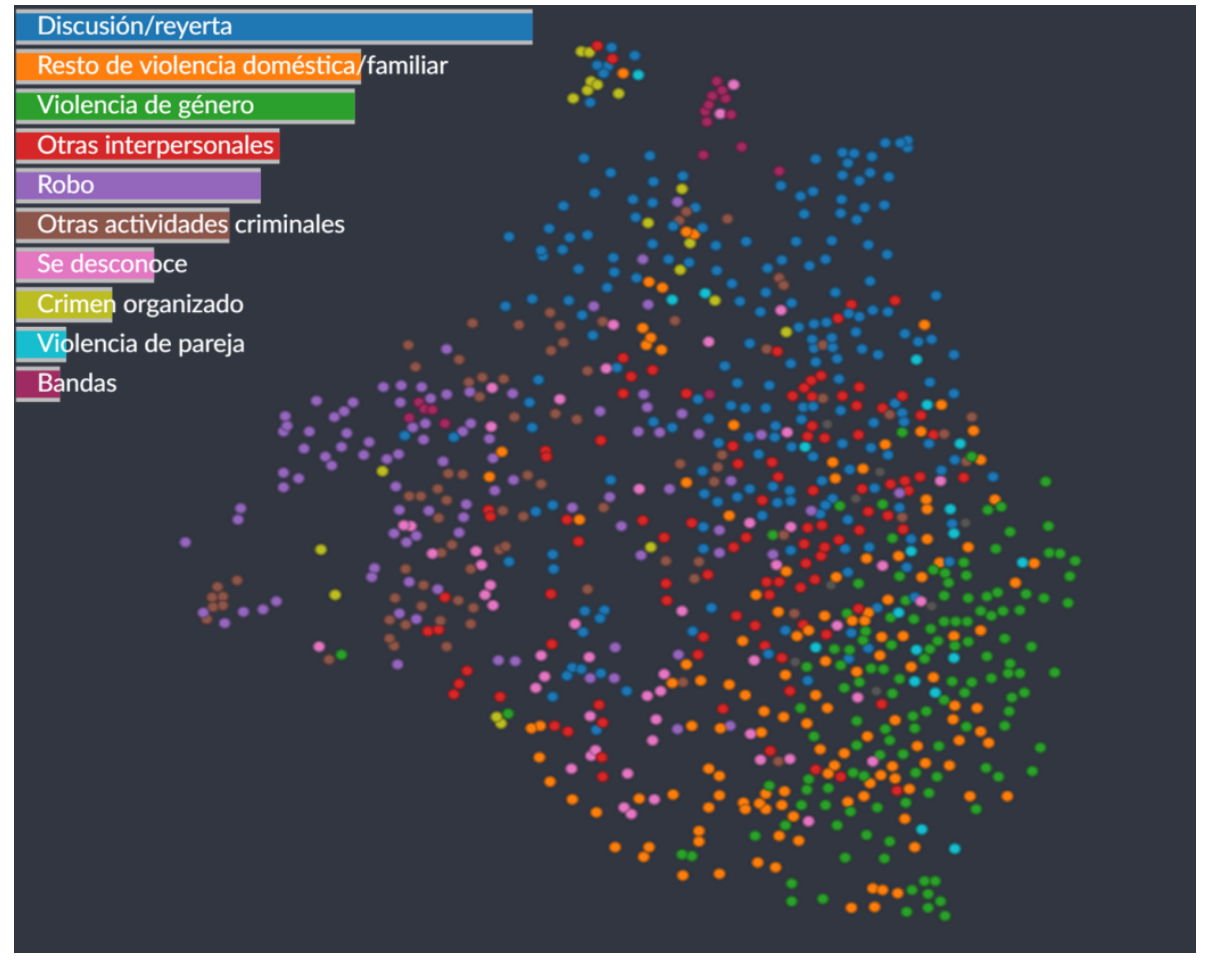

Figura 4. Casos coincidentes (variables de filtro: sexo = varón; edad = menor de edad).

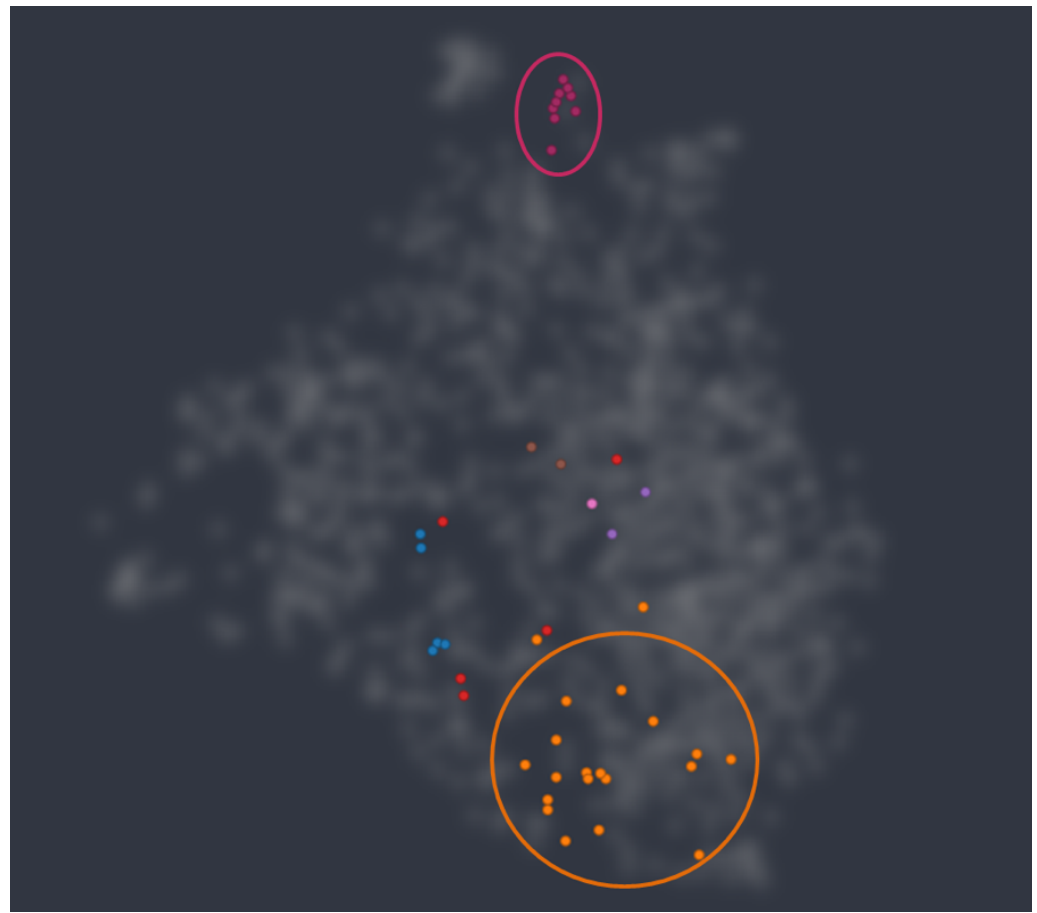

Una vez identificados los casos coincidentes, se comprobará el perfil prototípico del autor de cada uno de los tipos de homicidios principales identificados por el programa. En el caso de los homicidios relacionados con el mundo delincuencial, se trataría de autores varones de entre 13 y 17 años, que no tenían relación previa con la víctima y que actuaron acompañados. En el caso de los homicidios relacionados con el ámbito familiar, destacarían las mujeres, madre de la víctima, entre las franjas de edad de 35 a 40 y 55 a 60 años. Las investigaciones policiales son procesos dinámicos, y en cualquier 
momento puede conocerse nueva información. De ser así, esa nueva información puede incluirse en la búsqueda de casos coincidentes, ayudando a decantarse por una de las líneas de investigación.

\section{Futuro de la policía predictiva}

En la empresa privada y en la comunidad empresarial, la información y la inteligencia son factores esenciales para crear ventajas competitivas (OSCE, 2017). En ese aspecto, la labor encaminada a la lucha contra la criminalidad no es diferente, puesto que la ventaja estratégica basada en el conocimiento incrementa su eficacia para prevenir delitos e incidentes relacionados con la seguridad, y desarticular redes y grupos delictivos. Pero no basta con poseer abundante información o conocimiento sino que es necesario tener un marco adecuado para gestionar ese conocimiento y aprovecharlo al máximo, así como para asegurar que los datos y la información son recopilados, procesados y utilizados en estricto cumplimiento de las leyes nacionales y las normas internacionales en materia de derechos humanos (OSCE, 2017).

En este trabajo se ha hecho mención a algunas de las aplicaciones más relevantes que se conocen sobre policía predictiva en España, y que han supuesto pasar de un enfoque reactivo a un enfoque prospectivo, proactivo y preventivo, buscando actuar antes de que se cometa el delito, o ayudar a esclarecer hechos pasados. Aunque el enfoque preventivo no es nuevo, ya que los estudios clásicos de la Escuela de Chicago (Park et al., 1967), basados en el concepto de ecología humana ya tenían como objetivo estudiar las relaciones espacio-temporales del individuo con su entorno, dando lugar a los primeros mapas del crimen, el avance que ha supuesto la policía predictiva está en: 1) el aumento en la cantidad de datos que se pueden gestionar; 2) la rapidez con la que se pueden analizar; 3) la existencia de estándares de trabajo que comparten los usuarios de un sistema concreto; y 4) la posibilidad de crear modelos estadísticos que son capaces de aprender por sí mismos.

La policía predictiva debe entenderse como un conjunto de herramientas de apoyo en la toma de decisiones de los investigadores policiales. Algunos de los problemas que han planteado los detractores de su aplicación, vendrían fundamentalmente de un mal uso de estas herramientas. Pese a las posibilidades que ofrecen los modelos predictivos, no se debe dejar de lado el conocimiento teórico (Chan y Bennett Moses, 2016), ya que los investigadores policiales deben dotar de contexto a los resultados ofrecidos por los algoritmos. Los modelos basados en IA son capaces de analizar datos, y tomar decisiones en base a los resultados obtenidos, pero en el ámbito del crimen, ni los sistemas predictivos ni la sociedad están aún preparados para dejar las decisiones enteramente en manos de estos modelos, por lo que actualmente, ni la confianza excesiva, ni el rechazo total de estas prácticas, son beneficiosos, ya que el uso de los datos en la toma de decisiones es el futuro de los cuerpos policiales.

Los dos ejemplos que se han analizado en profundidad son aplicaciones de la policía predictiva, y de las técnicas preventivas, en eventos de baja prevalencia. Tanto los feminicidios, como los homicidios en general, son delitos que despiertan una gran alarma social, pero que tienen una serie de características que los hacen especialmente difíciles de predecir y analizar de forma sistemática (Berk y Sorenson, 2019). Pero precisamente se han empleado estos dos ejemplos por ser representativos de dos de los principales ámbitos de aplicación de la policía predictiva: estimar la probabilidad que tiene un individuo concreto de cometer un crimen, y predecir las características que, con mayor probabilidad, tendrá el autor de un crimen sin esclarecer.

Otro de los desafíos que debe afrontar la policía predictiva en los próximos tiempos, está relacionado con su implementación. En primer lugar, debe entenderse que no basta con crear programas o algoritmos y aplicarlos sobre una gran cantidad de datos, sino que debe haber un proceso de asimilación por parte de los usuarios policiales. Estos deben familiarizarse con las herramientas, capacitarse, extender la cultura analítica prospectiva, y, sobre todo, tienen ver que su uso facilitará su labor y aumentará su eficiencia. Si los investigadores policiales no ven útiles las herramientas, no entienden los resultados, o hacen un mal uso de estos sistemas, no se crearán estrategias eficientes, y esto revertirá en una pérdida de confianza por parte del usuario. Pero también es importante la aceptación social de estas herramientas. En parte, la desconfianza que pueden generar los modelos predictivos viene explicada por una mala concepción de lo qué son, de cómo funcionan, y de cuándo se aplican. Lo que propone la policía predictiva es dar un uso a los datos que hasta ahora había sido imposible, principalmente por falta de medios técnicos. Lo que se pretende es aprovechar al máximo el potencial de las bases de datos policiales, y no limitar el objetivo de la recogida sistemática de información a acreditar el cumplimiento de los requerimientos de la fase de investigación criminal.

A lo largo de todo el trabajo se ha incidido sobre la idea de que valorar el riesgo de que suceda un evento futuro no implica ni determina que esos eventos se vayan a producir. Los indicadores de riesgo, y todas las variables que analizan los sistemas 
predictivos, sirven para encontrar correlaciones, no causalidades. Otra idea importante que se ha intentado transmitir, es que ni la tecnología informática ni la estadística pueden suplantar, al menos de momento, al analista criminal. Deben ser las personas las que decidan sobre la aplicación que se hace de los resultados obtenidos de los análisis. Por otro lado, cabe destacar que no es necesario hacer grandes inversiones en software ni equipo informático, sino que lo que hay que promover son los estudios científicos del crimen. Además, se debe dar formación especializada a los investigadores policiales en el uso de este tipo de técnicas predictivas, ya que de nada sirve que se realicen análisis predictivos si estos no van acompañados de medidas operativas y estrategias policiales.

\section{Referencias}

Bennett Moses, L. y Chan, J. (2016). Algorithmic prediction in policing: assumptions, evaluation and accountability. Policing and Society, 28, 806-822. https://doi.org/10.1080/10439463.2016.1253695

Berk, R. A. y Sorenson, S. B. (2019). Algorithmic approach to forecasting rare violent events. An illustration based in intimate partner violence perpetration. Criminology \& Public Policy, 19(1), 213-233. https://doi.org/10.1111/1745-9133.12476

Fernández, C. (2019). Valoración del riesgo del radicalismo violento en el medio penitenciario. En R. Bermejo e I. Bazaga (Eds.), Radicalización violenta en España. Detección, gestión y respuesta, (pp. 253-259). Tirant Lo Blanch.

Bland, M. (2020). Algorithms Can Predict Domestic Abuse, But Should We Let Them? En H. Jahankhani, B. Akhgar, P. Cochrane y M. Dastbaz (Eds.), Policing in the Era of AI and Smart Societies (pp. 139-155). Springer.

Camacho-Collados, M. (2016). Statistical analysis of spatiotemporal crime patterns. optimization of patrolling strategies. (Tesis doctoral). Universidad de Granada, España.

Camacho-Collados, M. y Liberatore, F. (2015). A Decision Support System for predictive police patrolling. Decision Support Systems, $\quad 75, \quad 25-37$. https://doi.org/10.1016/j.dss.2015.04.012

Camacho-Collados, M., Liberatore, F. y Angulo, J. M. (2015). A multi-criteria Police Districting Problem for the efficient and effective design of patrol sector. European Journal of Operational Research, 246(2), 674-684. https://doi.org/10.1016/j.ejor.2015.05.023

Campbell, J. C. (2012). Risk Factors for Intimate Partner Homicide: The importance of Margo Wilson's Foundational research. Homicide Studies, 16, 438-444. https://doi.org/10.1177/1088767912463208

Canter, D. (2004). Offender profiling and Investigative Psychology. Journal of Investigative Psychology and Offender Profiling, 1, 1-15. https://doi.org/10.1002/jip.7
Chan, J. y Bennett Moses, L. (2016). Is big data challenging criminology? Theoretical Criminology, 20(1), 21-39. https://doi.org/10.1177/1362480615586614

Datta, A., Sen, S. y Zick, Y. (2016). Algorithmic transparency via quantitative input influence: Theory and experiments, with learning systems. In 2016 IEEE symposium on security and privacy. https://doi.org/10.1109/SP.2016.42

Dayan, K., Fox, S. y Morag, M. (2013). Validation of Spourse Violence Risk Assessment Inventory for Police Purposes. Journal of Family Violence, 28(8), 811821. https://doi.org/10.1007/s10896-013-9547-4

Delgado, R., González, J. L., Sotoca, A. y Tibau, X. A. (2016). Perfil del incendiario forestal: un Sistema Experto basado en redes bayesianas. Comunicación presentada al XI Congreso Español de Criminología. Abriendo vías a la reinserción.

Delgado, R., González, J. L., Sotoca, A. y Tibau, X. A. (2018). Archetypes of Wildfire Arsonists: An Approach by Using Bayesian Networks. IntechOpen. https://doi.org/10.5772/intechopen.72615

Eck, J. Chainey, S., Cameron, J., Leitner, M. y Wilson, R. (2005). Mapping Crime: Understanding Hot Spot. National Institute of Justice.

Ferguson, A. G. (2011). Crime Mapping and the Fourth Amendment: Redrawing "High-Crime Areas". Hastings Law Journal, 63(1), 179-232.

Ferguson, A. G. (2015). Big data and predictive reasonable suspicion. University of Pennsylvania Law Review, 163(2), 327-410.

García-Barceló, N., González-Álvarez, J. L., Almond, L. y Woolnough, P. (2020). Behavioural themes in Spanish missing person cases: An empirical typology. Journal of Investigative Psychology and Offender Profiling. Ahead of print. https://doi.org/10.1002/jip.1562

García-Barceló, N., Tourís-López R. M. y GonzálezÁlvarez, J. L. (2019). Personas desaparecidas: conveniencia de fomentar la investigación científica en España. Boletín Criminológico, 183.

Giménez-Salinas, A., Pérez, M., Vozmediano, L., San Juan, C., Ramos, D., González-Álvarez, J. L., Soto, J. E., Pozuelo, L. y De Juan, M. (2018). Agresores con victima desconocida. Implicaciones para la investigación criminal. Ministerio del Interior.

González, J. L. (2011). El perfil psicosocial del incendiario forestal. Boletín de la Asociación de Técnicos de Instituciones Penitenciarias, 25, 4-10.

González, J. L. (2015). La psicología criminalista en España: presente y futuro. Papeles del Psicólogo, 36(2), 109-116.

González, J. L., Garrido, M. J., López-Ossorio, J. J., Muñoz, J. M., Arribas, A., Carbajosa, P. y Ballano, E. (2018). Revisión pormenorizada de homicidios de mujeres en las relaciones de pareja en España. Anuario 
de Psicología Jurídica, 28, 28-38. https://doi.org/10.5093/apj2018a2

González, J. L., Muñoz, V., Calcerrada, M. L. y Sotoca, A. (2017). Perfil psicosocial del incendiario forestal español privado de libertad. Behavior \& Law Journal, 3(1), 26-34

González, J. L., Sánchez, F., López-Ossorio, J. J., Santos, J. y Cereceda, J. (2018). Informe sobre el homicidio. España 2010-2012. Ministerio del Interior. https://doi.org/10.13140/RG.2.2.18218.67526

González, J.L., Sotoca, A., Martín, M.J., Rodríguez, R., Rosique, M. T. y Martínez, J.M. (2010). Aplicación práctica del perfilamiento criminal: perfil psicosocial del incendiario forestal. En Jiménez, J. (2010). Manual Práctico del Perfil Criminológico. 345-370. Lex-Nova.

González-Álvarez, J. L., López-Ossorio, J. J. y MuñozRivas, M. (2018). La valoración policial del riesgo de violencia contra la mujer pareja en España - Sistema VioGén. Ministerio del Interior, Gobierno de España.

Gracia, E. (2004). Unreported cases of domestic violence against women: towards an epidemiology of social silence, tolerance, and inhibition. Journal of Epidemiology and Community Health, 58(7), 536-537. https://doi.org/10.1136/jech.2003.019604

Hardyns, W. y Rummens, A. (2017). Predictive Policing as a New Tool for Law Enforcement? Recent Developments and Challenges. European Journal on Criminal Policy and Research, 24(3), 201-218. https://doi.org/10.1007/s10610-017-9361-2

Hart, N. y Yohannes, M. (2019). Evidence Works: Cases Where Evidence Meaningfully Informed Policy. Bipartisan Policy Center.

Hilton Z., Harris, G., Rice M., Houghton R. y Eke, A. (2008). An Indepth Actuarial Assessment for Wife Assault Recidivism: The Domestic Violence Risk Appraisal Guide. Law and Human Behavior, 32(2), 150163. https://doi.org/10.1007/s10979-007-9088-6

Joh, E. E. (2014). Policing by numbers: big data and the fourth amendment. Washington Law Review, 89(35), 3568.

Kalcsics, J. Nickel, S. y Schöder. (2005). Towards a unified territorial design approach. Applications, algorithms and GIS integration. TOP: An Official Journal of the Spanish Society of Statistics and Operations Research, 13(1), 156. https://doi.org/10.1007/BF02578982

Kaufmann, M., Egbert, S. y Leese, M. (2019). Predictive policing and the politics of patterns. British journal of criminology, 59(3), 674-692. https://doi.org/10.1093/bjc/azy060

Kitchin, R. (2014). The Data Revolution: Big Data, Open Data, Data Infrastructures \& Their Consequences. Sage.

Koss, K. K. (2015). Leveraging predictive policing algorithms to restore fourth amendment protections in high-crime areas in a post-Wardlow world. Chicago-Kent Law Review, 90(1): 301-334.
Kropp, P. R. y Hart, S. D. (200). The Spousal Assault Risk Assessment (SARA) Guide: Reliability and Validity in Adult Male Offenders. Law and Human Behavior, 24, 101-118. https://doi.org/10.1023/A:1005430904495

Ley Orgánica 1/2004, de 28 de diciembre, de Medidas de Protección Integral contra la Violencia de Género. Boletin Oficial del Estado, 313.

Liberatore, F. y Camacho-Collados, M. (2016). A Comparison of Local Search Methods for the Multicriteria Police Districting Problem on Graph. Mathematical Problems in Engineering, vol. 2016.

Liberatore, F., Quijano-Sánchez, L. y Camacho-Collados, M. (2018). Applications of Data Science in Policing: VeriPol as an Investigation Support Tool. European Law Enforcement Research Bulletin, 4, 89-96.

López-Ossorio, J. J., Carbajosa, P., Cerezo-Domínguez, A. I., González-Álvarez, J. L., Loinaz, I. y Muñoz-Vicente, J. M. (2018). Taxonomía de los homicidios de mujeres en las relaciones de pareja. Psychosocial Intervention, 27(2), 95-104

López-Ossorio, J. J, González, J. L., Buquerín, S., García, L. F. y Buela-Casal, G. (2017). Risk Factors related to intimate partner violence police recidivism in Spain. International Journal of Clinical and Health Psychology, 17, 107-119

López-Ossorio, J. J., González-Álvarez, J. L., MuñozVicente, J. M., Urruela, C. y Andrés-Pueyo, A. (2019). Validation and Calibration of the Spanish Police Intimate Partner Violence Risk Assessment System (VioGén). Journal of Police and Criminal Psychology. https://doi.org/10.1007/s11896-019-09322-9

López-Ossorio, J. J., Loinaz, I. y González-Álvarez, J. L. (2019). Protocolo para la valoración policial del riesgo de violencia de género (VPR4.0): revisión de su funcionamiento. Revista española de Medicina Legal. https://doi.org/10.1016/j.reml.2019.01.002

López-Ossorio, J. J., González-Álvarez, J. L., Loinaz, I., Martínez-Martínez, A. y Pineda, D. (2020). Intimate partner homicide risk assessment by police in Spain: The Dual Protocol VPR $5.0-\mathrm{H}$. Psychosocial Intervention. https://doi.org/10.5093/pi2020a16

Macnish, K., Wright, D. y Jiya, T. (2020). Predictive Policing in 2025: A Scenario. En H. Jahankhani, B. Akhgar, P. Cochrane y M. Dastbaz (Eds.), Policing in the Era of AI and Smart Societies (pp. 139-155). Springer.

Delegación del Gobierno contra la Violencia de Género. (2020). Macroencuesta de Violencia contra la Mujer 2019.

Matias, A., Gonçalves, M., Soeiro, C. y Matos, M. (2019). Intimate partner homicide: A meta-analysis of risk factors. Aggression and Violent Behavior, 50. https://doi.org/10.1016/j.avb.2019.101358

Mitchell, P. S. (1972). Optimal Selection of Police Patrol Beats. The Journal of Criminal Law, Criminology, and Police Science, 63(4), 577-584. https://doi.org/10.2307/1141814 
Narayan, A. K., Lopez, D. B., Miles, R. C., Dontchos, B., Flores, E. J., Glover, M. y Lehman, C. D. (2019). Implementation of an Intimate Partner Violence Screening Assessment and Referral System in an Academic Women's Imaging Department. Journal of the American College of Radiology, 16(4), 631-634. https://doi.org/10.1016/j.jacr.2018.12.026

Organización Mundial de la Salud (OMS). (2013). Global and regional estimates of violence against women: prevalence and bealth effects of intimate partner violence and non-partner sexual violence.

Organización para la Seguridad y la Cooperación en Europa (OSCE). (2017). Guía de la OSCE sobre actividad policial basada en la inteligencia.

Oswald, M., Grace, J., Urwin, S. y Barnes, G. C. (2018). Algorithmic risk assessment policing models: lessons from the Durham HART model and 'experimental' proportionality. Inf Commun Technol Law, 27(2):223-250.

Park, R. E., Burgess, E. W. y McKenzie, R. D. (1967). The City. University of Chicago Press.

Perrot, P. (2017). What about AI in criminal intelligence? From predictive policing to AI perspectives. European Law Enforcement Research Bulletin, 16, 65-75.

Perry, W. L., McInnis, B., Price, C. C., Smith, S. C. y Hollywood, J. S. (2013). Predictive policing: The role of crime forecasting in law enforcement operations. Rand Corporation.

Portal Estadístico. Hechos conocidos. Madrid: Ministerio del Interior.

Provost, F. y Fawcett, T. (2013). Data Science for Business: What you need to know about data mining and data-analytic thinking. O'Reilly Media.

Quijano-Sánchez, L., Liberatore, F., Camacho-Collados, J. y Camacho-Collados, M. (2018). Applying automatic text-based detection of deceptive language to police reports: Extracting behavioral patterns from a multistep classification model to understand how we lie to the police. Knowledge-Based Systems, 149, 155-168.

Ratcliffe, J. H. (2016). Intelligence-Led Policing, (2 ${ }^{a}$ edición). Routledge.

Santos, J. y González, J. L. (2017). Homicidio de pareja en España (2007-2016): tiempo entre denuncia, valoración policial del riesgo y muerte. Behavior and Law Journal, $3(1), 1-10$.

Santos, J., González, J. L. y Touza, J. M. (2019). Homicidio en demarcación de la guardia civil. El uso de los datos en la investigación criminal. Cuadernos de la Guardia Civil, 59.

Schlehahn, E., Aichroth, P., Mann, S., Schreiner, R., Lang, U., Shepherd, I. D. y Wong, B. W. (2015). Benefits and pitfalls of predictive policing. In Intelligence and Security Informatics Conference (EISIC).

Seigfried-Spellar, K. C., Rogers, M. K., Rayz, J. T., Yang, S., Misra, K. y Ringenberg, T. (2019). Chat Analysis Triage Tool: Differentiating contact-driven vs. fantasy- driven child sex offenders. Forensic Science International. https://doi.org/10.1016/j.forsciint.2019.02.028

Sotoca, A., González, J. L., Fernández, S., Kessel, D., Montesinos, O. y Ruiz, M. (2013). Perfil del incendiario forestal español: aplicación del perfilamiento criminal inductivo. Anuario de Psicología Jurídica. 23, 31-38.

Spencer, C. M. y Stith, S. M. (2018). Risk Factors for Male Perpetration and Female Victimization of Intimate Partner Homicide: A Meta-Analysis. Trauma, Violence, \& Abuse, 21(3), 527-540. https://doi.org/10.1177/1524838018781101

Stöckl, H., Devries, K., Rotstein, A., Abrahams, N., Campbell, J., Watts, C. y García, C. (2013). The global prevalence of intimate partner homicide: a systematic review. The Lancet, 382, 859-865. https://doi.org/10.1016/S0140-6736(13)61030-2

Stockman, J. K., Lucea, M. B. y Campbell, J. C. (2013). Forced Sexual Initiation, Sexual Intimate Partner Violence and HIV Risk in Women: A Global Review of the Literature. AIDS and Behavior, 17, 832-847. https://doi.org/10.1007/s10461-012-0361-4

Strikwerda, L. (2020). Predictive policing: The risks associated with risk assessment. The Police Journal: Theory, Practice and Principles, 1-15. https://doi.org/10.1177/0032258X20947749

Tayebi, M. A. y Glässer, U. (2016). Social network analysis in predictive policing: Concepts, models and methods. Springer.

Townsley, M. (2017). Crime mapping and spatial analysis. En B. Leclerc y E. Savona (Eds.), Crime prevention in the $21^{\text {st }}$ century (pp. 101-112). Springer International Publishing.

Wang, F. (2012). Why police and policing need GIS: an overview. Annals of GIS, 18(3), 159-171. https://doi.org/10.1080/19475683.2012.691900

Waske, B. y Beneditksson, J. A. (2014). Pattern Recognition and Classification. En E. G. Bjoku (Ed.), Encyclopedia of Remote Sensing (pp. 503-23). Springer. 